\section{Seroprevalencia de hantavirus en roedores y casos humanos en el sur de la Argentina}

\section{Hantavirus seroprevalence in rodents and human cases in southern Argentina}

\section{Edmundo Larrieu \\ Faculdad de Ciencias Veterinarias Universidad Nacional de la Pampa Secretaria de Estado de Salud Provincia de Rio Negro Laprida 240 (8500) Viedma, Argentina elarrieu@salud.rionegro.gov.ar \\ Eduardo Herrero \\ Secretaria de Estado de Salud Provincia de Rio Negro \\ Mariela Garcia Cachau \\ Faculdad de Ciencias Veterinarias Universidad Nacional de la Pampa \\ Jose Luis Labanchi \\ Secretaria de Estado de Salud \\ Provincia de Rio Negro \\ Sergio Mancini \\ Secretaria de Estado de Salud \\ Provincia de Rio Negro \\ Paula Padula}

Instituto Nacional de Enfermedades Infecciosas INEI - Anlis “Dr. C.G. Malbrán”

\section{Gustavo Cantoni}

Secretaria de Estado de Salud

Provincia de Rio Negro

\section{Laura Cavagion}

Faculdad de Ciencias Veterinarias Universidad Nacional de la Pampa

\section{Emiliano Alvarez}

Faculdad de Ciencias Veterinarias Universidad Nacional de la Pampa

\section{Maria Bruni}

Faculdad de Ciencias Veterinarias Universidad Nacional de la Pampa

Silvina Albarracin

Secretaria de Estado de Salud Provincia de Rio Negro

\section{Odila Arellano}

Secretaria de Estado de Salud Provincia de Rio Negro

\section{Resumen}

En la Provincia de Río Negro, Argentina, se presentaron casos humanos de síndrome pulmonar por hantavirus (SPH) en la región de la cordillera andino patagónica. El virus Andes ha sido identificado en la región, tanto en el roedor Oligoryzomys longicaudatus como en seres humanos, demostrándose la transmisión principalmente del roedor al hombre y la factibilidad de la transmisión de persona a persona.

El objetivo del presente trabajo es presentar nueva información sobre especies de roedores portadores de hantavirus en Argentina, su prevalencia de anticuerpos para hantavirus (período 1999-2001) y la relación del tamaño de las poblaciones de roedores y su seroprevalencia con la ocurrencia de casos humanos (período 1996-2001).

Para ello, se procedió a la colocación de 3973 trampas para captura viva de roedores, tipo sherman en seis operativos efectuados entre octubre de 1999 y mayo de 2001. Se obtuvieron muestras de sangre de los roedores las que fueron procesadas mediante enzimoinmunoensayo con antígenos elaborados a partir de virus Andes. Una síntesis de los resultados indica 397 roedores capturados, con un éxito de trampeo del $10 \%$ y una prevalencia de anticuerpos contra hantavirus del $1.0 \%$. Se observaron importantes diferencias en las especies capturadas en cada una de las regiones. Se capturaron $O$. longicaudatus y A. Olivaceus seropositivos y $O$. flavescens y C. Laucha potencialmente portadores de hantavirus

Se registraron 6 casos humanos en el período 1993-1995 (correspondientes a estudios retrospectivos), 21 casos se notificaron en el período 1996-1998 y 6 en el período 1999-2001

Se analiza la correlación entre ocurrencia de casos humanos, seroprevalencia en roedores y éxito de trampeo.

Palabras Claves: Hantavirus. Epidemiologia. Roedores. Sindrome Pulmonar. 
In the Province of Río Negro, Argentina, human cases of hantavirus pulmonary syndrome (HPS) have occurred in the region of the Patagonian Andean range. The Andes virus has been identified in the region, both in the rodent Oligoryzomys longicaudatus and in human beings, demonstrating mainly transmission from rodents to human and the possibility of person-to-person transmission.

The goal of this paper is to present new information on hantavirus rodent carrier species in Argentina, the prevalence of antibodies to hantavirus (1999-2001 period) and the relationship of the rodent population size and seroprevalence with the occurrence of human cases (1996-2001 period).

To this end, a total of 3,973 Sherman type traps for capturing live rodents were placed in six campaigns from October 1999 to May 2001. Rodent blood samples were obtained and processed by means of enzymoimmunoassay with antigens developed from the Andes virus. A summary of results indicates 397 captured rodents, with a $10 \%$ trapping success rate and a $1.0 \%$ prevalence of antibodies to hantavirus. Considerable differences were observed in species captured in each region. Seropositive $O$. longicaudatus and A. olivaceus specimens, as well as potential hantavirus $O$. flavescens and $C$. laucha carriers, were captured.

Six human cases were recorded during the 1993-1995 period (corresponding to retrospective studies), while 21 cases were reported in 1996-1998 and 6 in 1999-2001.

The correlation between occurrence of human cases, seroprevalence in rodents and trapping success is analyzed.

Key Words: Hantavirus. Epidemiology. Rodents. Pulmonary syndrome.

\section{Introducción}

El prototipo del género Hantavirus, familia Bunyanviridae, fue aislado en Corea en la década de 1970 y se denominó virus Hantaan. Desde entonces a todos los virus relacionados se les conoce como Hantavirus. Todas las enfermedades causadas por estos virus se agruparon con el nombre de Fiebre Hemorrágica con Síndrome Renal (FHSR).

A partir de 1993 se describen una serie de muertes por neumopatía fulminante entre población indígena de la región de Four Corners (Estados norteamericanos de Nuevo México, Arizona, Colorado y Utha), identificándose como agente etiológico una nueva especie de Hantavirus denominado Virus Sin Nombre (SNV). La enfermedad, de una letalidad que alcanzó al $52 \%$, fue designada síndrome pulmonar por hantavirus (SPH) ${ }^{1}$.

El primer reservorio para SPH fue identificado en EEUU en 1994 y correspondió a un roedor silvestre, Peromyscus maniculatus $^{2}$. Posteriormente se han identificado otros reservorios tal como Sigmodon hispidus, Oryzomis palustris y Peromyscus leucopus (EEUU), Calomys laucha (Paraguay y Bolivia), Oligoryzomis microtis (Peru), Oligoryzomis fulvescens (Panama) y Oligoryzomys longicaudatus y Oligoryzomys flavescens (Argentina). Todos los reservorios corresponden a la familia Muridae, subfamilia Sigmodontinae ${ }^{2-12}$.

Durante el período 1996-2001 la Dirección Nacional de Epidemiología dependiente del Ministerio de Salud de Argentina ${ }^{13}$ informó 374 casos, resultando las provincias mas afectadas Salta (111 casos), Buenos Aires (104 casos), Jujuy (58 casos), Río Negro (27 casos), Santa Fe (20 casos), Chubut (18 casos) y Neuquen (17 casos). La letalidad alcanzó al 30\%. Todos los casos han estado asociados a alguno de los cinco linajes de hantavirus Andes: AND Nort, AND Sout, AND Cent Lec, AND Cent Bs As, AND Cent Plata ${ }^{14}$.

En la Provincia de Río Negro en particular, todos los casos ocurrieron en la región de la cordillera andino patagónica, estando siempre asociados a virus Andes (linaje AND Sout) trasmitido desde Oligoryzomys 
longicaudatus infectados ${ }^{15} \mathrm{o}$ de persona a persona $^{16,17}$.

Estudios sucesivos han sido efectuados en la región de la cordillera andino patagónica de esta Provincia con el objetivo de describir brotes ${ }^{18}$, evaluar factores de riesgo $^{19}$, evaluar aspectos clínicos de la ocurrencia $^{20}$ y estimar la prevalencia de anticuerpos para hantavirus en roedores ${ }^{15,18}$. En la Provincia de La Pampa, por su parte, no se han notificado casos de SPH.

Así, el objetivo del presente trabajo es presentar nueva información sobre especies de roedores portadores de hantavirus en Argentina, su prevalencia de anticuerpos para hantavirus (período 1999-2001) y la relación del tamaño de las poblaciones de roedores y su seroprevalencia con la ocurrencia de casos humanos (período 1996-2001).

\section{Materiales y Métodos}

\section{Area de trabajo}

- Con ocurrencia de casos humanos (cordillera andino patagónica):

Departamento Bariloche al oeste de la Provincia de Río Negro, Argentina, en la cordillera andino-patagónica. El clima es frío y húmedo, con isohietas de $800 \mathrm{~mm}$ a 1000 $\mathrm{mm}$. La vegetación es frondosa, coexistiendo bosques densos con áreas con predominio de vegetación arbustiva (Rose rubiginosa, Rubus idaneus, etc). Los sistemas de producción agrícola incluyen hongos, frutillas, lúpulo y frutas finas.

- Sin ocurrencia de casos humanos:

Estepa patagónica: Departamentos Pilcaniyeu y San Antonio, ubicados en los extremos este y oeste respectivamente de la estepa patagónica en la Provincia de Río Negro. El clima es frío en invierno y caluroso en verano, con isohietas de $200 \mathrm{~mm}$ a 400 mm. La vegetación es escasa, con predominio de especies arbustivas, xerófilas y un mínimo de estrato herbáceo.

Pradera pampeana: Departamentos Maracó y Trenel, al noroeste de la Provincia de La Pampa. Su clima es templado y húmedo. La vegetación comprende tierras cultivadas con trigo, maíz y girasol, praderas para pastoreo de ganado y pequeños bosques de caldén (Prosopis caldenia).

\section{Trabajo de campo}

Se procedió a la captura de roedores mediante la instalación de líneas de trampas tipo Sherman (captura viva) con avena como cebo. Cada línea estuvo compuesta por $10 \mathrm{a}$ 25 trampas ubicadas cada 2 metros aproximadamente una de otra.. Las líneas fueron dispuestas de forma tal de abarcar la mayor variedad posible de estratos herbáceos y arbustivos, arroyos y cursos de agua y edificaciones rurales favorables a la presencia de roedores. Se aplicaron procedimientos estandarizados tanto para la colocación como para bioseguridad de los operadores $^{21}$.

Se efectuaron seis operativos entre octubre de 1999 y mayo de 2001 correspondiendo dos a cada región. En la cordillera andino patagónica se colocaron 671 trampas, en la estepa patagónica 1318 trampas y en la región pampeana 1984 trampas (total 3973 trampas).

Los roedores capturados fueron anestesiados mediante inhalación con éter o con metoxifluorano, obteniéndose muestras de sangre mediante punción cardíaca. Posteriormente los roedores fueron sacrificados mediante dislocación cervical y necropsiados para obtención de hígado, pulmón, bazo y riñón. Todas las muestras fueron conservadas en nitrógeno líquido hasta su procesado en el laboratorio del Instituto Nacional de Enfermedades Infecciosas INEI-Anlis "Dr. C. G. Malbrán”.

\section{Análisis de laboratorio}

Las muestras de sangre fueron procesadas mediante enzimoinmunoensayo (EIE) con antígenos elaborados a partir de virus Andes ${ }^{22}$. 
Análisis de los antecedentes epidemiológicos en el área con ocurrencia de casos

La Provincia de Río Negro mantiene un sistema de vigilancia de hantavirus que incluye:

- Determinación de la prevalencia de anticuerpos contra hantavirus en roedores en distintos momentos del año y estimación de las variaciones en el tamaño de sus poblaciones, información obtenida en 7 operativos de campo en el área de la cordillera andino patagónica efectuados en el período 1996-1999, cuyos resultados son publicados separadamente ${ }^{15}$ y los 2 efectuados en el presente trabajo, todos con similar metodología de campo.

- Registro de casos humanos en el período 1993-2001

Con esta información se analizó estadísticamente la relación entre las variables población de roedores (expresada como éxito de trampeo o porcentaje de roedores capturados por cada 100 trampas colocadas), prevalencia de anticuerpos contra hantavirus en roedores (porcentaje de roedores seropositivos) y ocurrencia de casos humanos (número de casos), mediante la estimación de los coeficientes de correlación de Pearson, suponiendo una distribución normal de las variables, efectuada con el software BMDP (SPSS, Chicago, IL).

\section{Resultados}

\section{Captura de roedores y determinación de prevalencia de anticuerpos contra hantavirus}

- Area con ocurrencia de casos humanos (cordillera andino patagónica):

En noviembre del 2000, se capturaron 28 roedores (éxito de trampeo 13.6\%), de los cuales 2 correspondientes a Oligoryzomys longicaudatus resultaron positivos (prevalencia total de anticuerpos contra hantavirus $7.1 \%$, en Oligoryzomis longicaudatus $18 \%$ ), siendo las especies capturadas Oligoryzomys longicaudatus $39.3 \%$, Abrothrix longipilis $35.6 \%$, Abrothrix olivaceus $14.3 \%$, Loxodontomus micropus3.6\%, Eligmodontia morgani3.6\%, Mus musculus 3.6\%.

En mayo del 2001, se capturaron 56 roedores, (éxito de trampeo 12\%) de los cuales 1 correspondiente a Oligoryzomys longicaudatus resultó positivo (prevalencia de anticuerpos contra hantavirus total $1.8 \%$, en Oligoryzomis longicaudatus 4.5\%). Las especies capturadas resultaron Oligoryzomys longicaudatus 39.3, Abrothrix longipilis $17.9 \%$, Abrothrix olivaceus $17.9 \%$, \%, Abrothrix xhantorin 8.9\%, Eligmodontia morgani 5.4\%, Mus musculus 5.4\%, Reitherodon auritus 3.6, otros 1.6\%.

- Area sin ocurrencia de casos humanos:

En el oeste de la región de la estepa patagónica, en abril del 2001, se capturaron 161 roedores (éxito de trampeo $35.3 \%$ ) de los cuales 1 correspondiente a Abrothrix olivaceus resultó positivo (prevalencia de anticuerpos contra hantavirus total $0.6 \%$, en Abrothrix olivaceus 3.1\%, en Oligoryzomis longicaudatus $0 \%$ ). Las especies capturadas resultaron Abrothrix xhantorin 27.9\%, Abrothrix olivaceus 20.5\%, Reitherodon aurirus $17.4 \%$, Oligoryzomys longicaudatus $11.2 \%$, Phyllotis xanthopygus $9.3 \%$, Abrothrix longipilis $8.1 \%$, Eligmodontia morgani $4.3 \%$ y Loxodontomys micropus $1.3 \%$.

En el este de la misma región, en mayo del 2001, se capturaron 20 roedores (éxito de trampeo $2.1 \%$, prevalencia de anticuerpos contra hantavirus total $0 \%$ ), resultando las especies capturadas Mus musculus $80 \%$, Reitherodon aurirus $10 \%$ y otros $10 \%$.

En la región de la pradera pampeana en octubre de 1999, se capturaron 47 roedores (éxito de trampeo $5.5 \%$, prevalencia de anticuerpos contra hantavirus total $0 \%$ ) siendo las especies capturadas Mus musculus $38.3 \%$, Akodon azarae $21.3 \%$, Akodon molinae $21.3 \%$, Calomys laucha6.4\% y Rattus ratus $4.2 \%$.

En la misma región, en noviembre del 2000 fueron capturados 87 roedores (éxito de trampeo $7.6 \%$, prevalencia de anticuerpos contra hantavirus total $0 \%$ ) siendo las especies capturadas Akodon molinae 48.3\%, 
Oligoryzomys flavescens 22.9\%, Akodon azarae $12.6 \%$, Calomys laucha $6.9 \%$, Mus musculus 3.4\%, Rattus ratus 3.4\%, Calomys musculinus $2.5 \%$.

En total se capturaron 397 roedores (éxito de trampeo $10 \%$ ), resultando la prevalencia de anticuerpos contra hantavirus del $1.0 \%$. (Tabla 1)

\section{Análisis de antecedentes}

Se registraron 6 casos en el período 19931995 (correspondientes a estudios retrospectivos), 21 casos se notificaron en el período 1996-1998 (13\% de los casos del país) y 6 en el período 1999-2001 (2.8\% de los casos del país).

Las variaciones en el tamaño de las poblaciones de roedores, la prevalencia de anticuerpos contra hantavirus en roedores en general y en Oligoryzomys longicaudatus en particular y la ocurrencia de casos humanos se presenta en tabla 2. La correlación de Pearson fue negativa para las variables éxito de trampeo/casos humanos (r: -0.2) y para la correlación éxito de trampeo/sero- prevalencia (r: -0.2) y positiva para las variables prevalencia de anticuerpos contra hantavirus/casos humanos (r: +0.2). En los tres casos resultó estadísticamente no significativa $(p>0.05)$.

\section{Discusion}

La presencia de $O$. longicaudatus positivos a anticuerpos contra hantavirus en la cordillera andino patagónica de la Provincia de Río Negro hallada en el presente trabajo, ha sido reportada en trabajos previos, confirmándose así su importancia como reservorio del virus Andes (linaje AND Sout. $)^{5,15,18}$.

En la estepa patagónica colindante fueron también capturados ejemplares de $O$. longicaudatus, aunque en baja proporción y negativos a anticuerpos contra hantavirus.

Por el contrario, no se capturaron ejemplares de $O$. longicaudatus en la región este de la estepa patagónica y en la pradera patagónica, en forma coincidente con la distribución territorial esperada de esta especie.

Tabla 1. Captura y prevalencia de anticuerpos contra hantavirus en roedores de las Provincias de Río Negro y La Pampa, Argentina, 1999-2001

Table 1. Capture and prevalence of antibodies to hantaviruses in rodents of Rio Negro and La Pampa Provinces. Argentina, 1999-2001

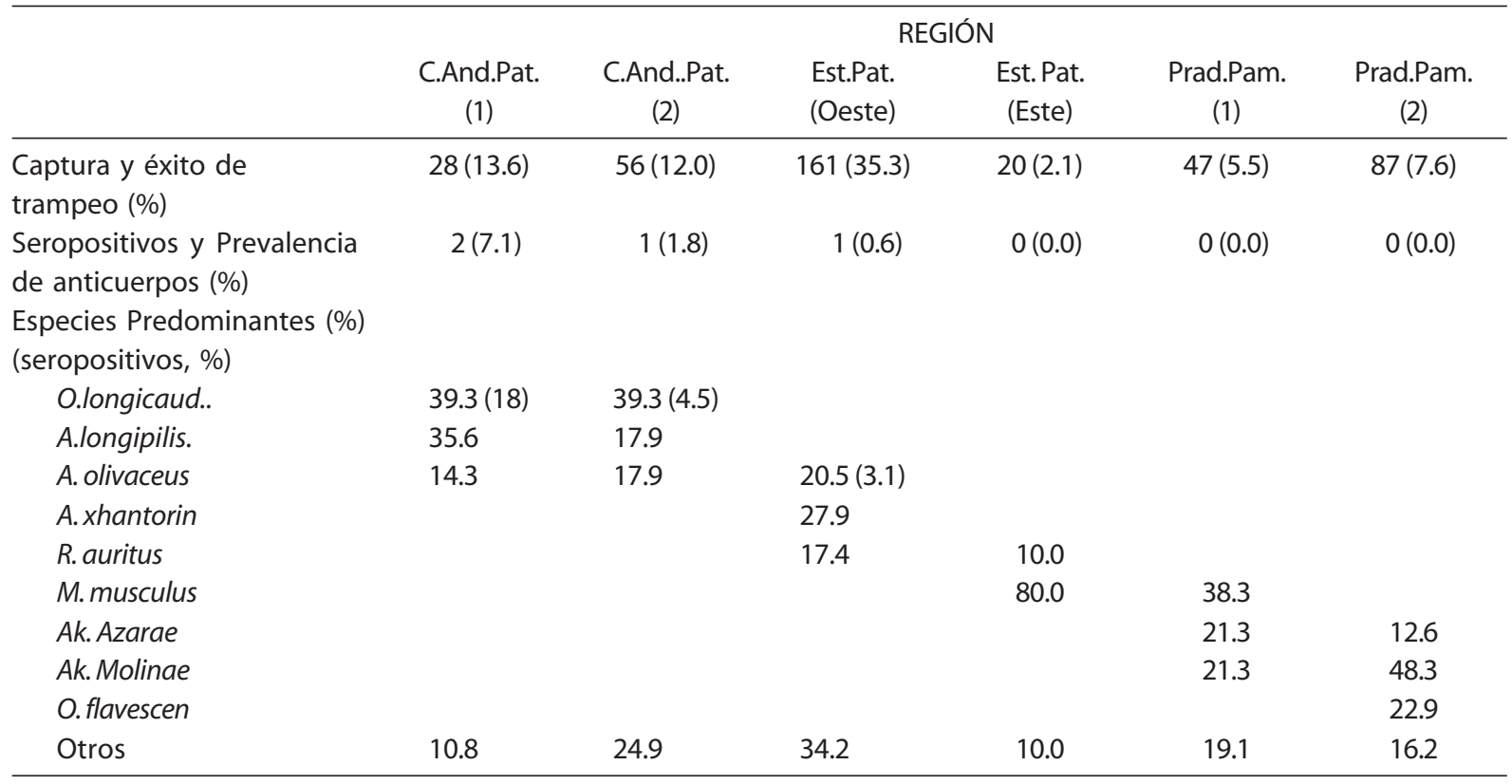


Tabla 2. Captura, prevalencia de anticuerpos contra hantavirus en roedores y casos humanos en la región de la cordillera andino patagónica, Provincia de Rio Negro, Argentina, 1996-2001

Table 2. Capture, prevalence of antibodies to hantaviruses in rodents and human cases, Rio Negro Province. Argentina, 1996-2001

\begin{tabular}{lcccc}
\hline Año & $\begin{array}{c}\text { Éxito de trampeo } \\
\text { de roedores (\%) }\end{array}$ & $\begin{array}{c}\text { Seroprevalencia en } \\
\text { roedores }\end{array}$ & $\begin{array}{c}\text { Seroprevalencia } \\
\text { en O.I. (\%) }\end{array}$ & Casos Humanos \\
\hline 1996 (enero) & 14.0 & 0 & 0 & 1 \\
1996 (octubre) & 13.2 & 7.5 & 13.8 & 10 \\
1996 (diciembre) & 2.7 & 5.7 & 9.3 & 7 \\
1997 (abril) & 8.3 & 1.5 & 2.1 & 0 \\
1997 (agosto) & 8.3 & 1.5 & 2.4 & 0 \\
1997 (octubre) & 90.6 & 1.5 & 2.1 & 0 \\
1997 (diciembre) & 10.0 & 16.6 & 33.3 & 1 \\
1998 & S/D & S/D & S/D & 1 \\
1999 & S/D & 7.1 & 18.0 & 1 \\
2000 (noviembre) & 13.6 & S/D & S/D & 3 \\
2000 & S/D & 0.7 & 1.6 & 1 \\
2001 (mayo) & 12.4 & & & \\
\hline
\end{tabular}

En la región de la estepa patagónica, se capturaron A. olivaceus positivos a anticuerpos contra hantavirus, lo que puede indicar la presencia de una especie potencialmente hospedadora de hantavirus patógenos para el hombre, situación previamente descripta $^{23}$ o representar pasajes de virus Andes del hospedador natural $O$. longicaudatus a otra especie de roedor con quién entra en contacto, situación que ha sido descripta en la región de la cordillera andino patagónica de la Provincia de Río Negro para A. longipilis y L. micropus hallados positivos a anticuerpos contra hantavirus aunque en muy baja proporción ${ }^{15}$.

En la región de la pradera pampeana, finalmente, se capturaron ejemplares de $O$. flavescens y C. laucha, serológicamente negativos. Sin embargo, estos roedores han sido notificados como reservorios de hantavirus patógenos para el hombre. O. flavescens como portador del virus Andes (linaje AND Cent. Lec.) fue asociado a casos en el nordeste de la Provincia de Buenos Aires en Argentina y $C$. laucha como portador del virus Laguna Negra se asoció a brotes ocurridos en Paraguay ${ }^{3,8,24}$.

La identificación de especies potencialmente portadoras de hantavirus y el hallazgo de nuevas especies seropositivas, aún en ausencia de casos humanos notificados, señalan la existencia de riesgos potenciales para las poblaciones humanas de la estepa patagónica y la pradera pampeana y requieren de la intensificación de medidas de vigilancia epidemiológica y de prevención.

En relación a los análisis de correlación, no se hallaron asociaciones estadísticamente significativas entre las variables estudiadas. La ocurrencia esporádica y ocasional de casos humanos de hantavirus ha limitado la posibilidad del análisis estadístico.

Sin embargo, se observa correlación positiva entre aumentos en la prevalencia contra anticuerpos contra hantavirus en roedores y la aparición de casos humanos, en especial con prevalencias de anticuerpos contra hantavirus en roedores cercanas o superiores al $10 \%$. La correlación entre aumento en las poblaciones de roedores y la aparición de casos humanos, por el contrario, muestra correlación negativa.

Si bien estos resultados son coincidentes con estudios previos efectuados en la región ${ }^{15}$, son necesarias mayores observaciones para establecer asociaciones estadísticas válidas.

Las variaciones temporales en la incidencia de la enfermedad en el hombre han sido correlacionadas con diversos factores 
ambientales. Para $O$. longicaudatus se ha notificado en Chile la ocurrencia de cambios en la prevalencia de la enfermedad debido a un brusco aumento en la disponibilidad de alimento para los roedores ${ }^{23,25}$, mientras en Estados Unidos, para P. maniculatus se ha encontrado una fuerte asociación entre aparición de casos humanos y el efecto meteorológico del $\mathrm{Niño}^{26}$.

Actualmente se proponen diversos modelos para la evaluación de modificaciones en los niveles de riesgo para hantavirus basados en el desarrollo de sistemas de vigilancia ambiental $(27,28)$ que posibiliten establecer medidas oportunas y racionales de prevención, educación sanitaria y control.

\section{Referencias}

1. Nichol ST, Spiropoulou CF, Morzunov S, Rollin PE, Ksiazek TG, Feldmann H. Genetic identification of a Hantavirus associated with an outbreak of acute respiratory illness. Science 1993; 5: 914-7.

2. Child JE, Ksiazek TG, Spiropoulou CF, Krebs JW, Morzunov S, Maupin GO. Serologic and Genetic identification of Peromiscus maniculatus as the primary reservoir for a new hantavirus in the southwestern United State. J infect Dis 1994; 169: 1271-80.

3. Martínez V P, Colavecchia S, García Alay M., Suzuki B, Trincheri A, Bustos S. Rabinovich R, Padula P. Sindrome Pulmonar por hantavirus en la Provincia de Buenos Aires. Medicina 2001; 61: 147-56.

4. Levis S, Rowe J, Morzunov S, Enría D, Jeor S. New hantaviruses causing hantavirus pulmonary syndrome in central argentina. Lancet 1997; 349: 9989 .

5. Calderon G, Pini N, Bolpe J, Levis S, Mills J, Segura E, Guthmann N, Cantoni G, Enria D, Hantavirus reservois host associated with peridomestic habitats in Argentina. Emer Infec Dis 1999; 5: 792-7.

6. Lopez N, Padula P, Rossi C, Lazaro M. Genetic identification of a new Hantavirus causing severe pulmonary syndrome in Argentina. Virology 1996; 6: 220-3.

7. Williams J, Bryan R, Mills J, Palma E, Vera I, Peters C, Zaki S, Khan A, Ksiazek T. Ann outbreak of hantavirus pulmonary syndrome in western Paraguay. Am J of Trop Med Hyg 1997; 57: 274-82.

8. Padula P, Colavecchia S, Martinez P, Gonzalez Della Valle M, Edelstein A, Miguel S, Russi J, Mora Riquelme J, Colucci N, Almiron M, Rabinovich D. Genetic diversity, distribution and serological features of Hantavirus infection in five Countries in South America. J Clin Microbiol 2000; 38: 3029-35.

9. Hjelle, B, Torrez Martinez, N, Koster, F. Hantavirus Pulmonary Syndrome-related virus from Bolivia. Lancet 1996; 347: 57.
10. Johnson A, Souza L, Ferreira I, Pererira L, Ksiazeck T, Rollin P, Peters C, Nichols S. Genetic investigation of novel hantaviruses causing fatal HPS in Brazil. J Med Virol 1999; 59: 527-35.

11. Powers A, Mercer D, Watts D, Guzman H, Fulhorst C, Popov V, Tesh R. Isolation and genetic characterization of a hantavirus from a rodent, Oligoryzomys microtis (Muridae), collected in northeastern Peru. Am J Trop Med Hyg 1999; 61: 92-8.

12. Vincent M, Quiroz E, Gracia F, Sanchez A, Ksazek T, Kitsutani P, Ruedas L, Tinnin D, Caceres L, Garcia A, Rollin P, Mills J, Peters C, Nichol S. Hantavirus pulmonary sindrome in Panama: Identification of novel hantaviruses and their likely reservoirs. Virology 2000; 277: 14-9.

13. Dirección Nacional de Epidemiología del Ministerio de Salud. Boletín Epidemiológico Nacional 1999.

14. Lopez N, Padula P, Rossi C, Miguel S, Edelstein A, Ramirez E. Genetic characterization and phylogeny of Andes virus and variants from Argentina and Chile. Virus Res 1997; 50: 77-84.

15. Cantoni G, Padula P, Calderón G, Mills J, Herrero E, Sandoval P, Martinez V, Pini N, Larrieu E. Seasonal variation in prevalence to hantaviruses in rodents from southern Argentina. Trop Med Int Health 2001; 6: 8116.

16. Enría D, Padula P, Segura E, Pini N, Edelstein A, Riva Posse C, Weissenbacher M. Hantavirus Pulmonary Syndrome in Argentina. Possibility of Person to Person transmission. Medicina (Buenos Aires) 1996; 56: 709-11

17. Padula P, Edelstein A, Miguel S, Lopez M, Rossi C, Rabinovich R. Hantavirus pulmonary syndrome outbreak in Argentina: molecular evidence for personto-person transmission of Andes virus. Virology 1998; 15: 323-30.

18. Cantoni G, Lázaro M, Resa A, Arellano O, Amestoy C, Herrero E, Perez A, Larrieu E (1997) Hantavirus pulmonary syndrome in the Province of Rio Negro, Argentina, 1993-1996. Rev Med Trop Sao Paulo 1997; 39: 191-6. 
19. Yadón, Z,. Epidemiología del síndrome pulmonar por hantavirus en la Argentina (1991-1997). Medicina (Buenos Aires) 1998; 58: 25-6.

20. Lazaro M, Resa A, Barclay C, Calanni L, Samengo L, Martinez L, Padula P, Pini N, Lasala M, Elsner B, Enría D. Sindrome pulmonar por hantavirus en el sur argentino. Medicina (Buenos Aires) 2000; 60: 289-301.

21. Mills J, Childs J, Ksiazek G, Peters C. Methods for trapping and sampling small mammals for virologic testing. Atlanta: U.S Department of Health and Human Services, Center for Disease Control and Prevention; 1995.

22. Padula P, Rossi C, Della Valle M, Martinez V, Colavecchia C, Edelstein A, Miguel D, Rabinovich D, Segura L. Development and evaluation of a solid phase enzyme immunoassay based on Andes hantavirus recombinant nucleoprotein. J Med Microb 2000; 49: 149-55.

23. Toro J, Vega J, Khan A, Mills J, Padula P. An outbreak of hantavirus pulmonary syndrome, Chile, 1997. Emerg Infect Dis 1998; 4: 687-94.

24. Johnson A, Bowen M, Ksiazek T, Williams R, Bryan R, Mills J, Peters C, Nichol S. Laguna negra virus associated with HPS in western Paraguay and Bolivia. Virology 1997; 10: 115-27.
25. Murua R, Gonzalez Le, Gonzalez M, Jofre Yc. Efectos del florecimiento del arbusto Chusquea quila Kunth (poaceae) sobre la demografía de poblaciones de roedores de los bosques templados fríos del sur chileno. Concepción, Chile: Boletín de la Sociedad de Biología, Concepción; 1996; 50: 77-84.

26. Hjjelle B, Glass G. Outbreak of hantavirus infection in the Four Corner region of the United States in the wake of the 1997-1998 El niño southern oscillation. J Infect Dis 2000; 181: 1569-73.

27. Boone J, McGwire K, Otteson E, DeBaca R, Kun E, Villard P, St Jeor S. Remote sensing and geographic information systems: charting Sin Nombre virus infection in deer mice. Emerg Infect Dis 2000; 6: 24858.

28. Glass G, Cheek J, Patz J, Shields T, Doyle T, Hunt D, Enscore R, Gage K, Irland C, Peters C, Bryan R. Using remotely sensed data to identify areas at risk for hantavirus pulmonary syndrome. Emerg Infect Dis 2000; 6: 238-47.

Recebido em: 11/03/02

$1^{\text {a }}$ aprovação em: 15/05/02

Versão final em: 21/06/02 